\title{
Are Nuclear Star Clusters the Precursors of Massive Black Holes?
}

\author{
Nadine Neumayer ${ }^{1,2}$ and C. Jakob Walcher ${ }^{3}$ \\ ${ }^{1}$ European Southern Observatory, Karl-Schwarzschild Straße 2, 85748 Garching bei München, Germany \\ ${ }^{2}$ Excellence Cluster Universe, Boltzmann Straße 2, 85748 Garching bei München, Germany \\ ${ }^{3}$ Leibniz-Institut für Astrophysik Potsdam (AIP), An der Sternwarte 16, 14482 Potsdam, Germany
}

Correspondence should be addressed to Nadine Neumayer, nneumaye@eso.org

Received 15 August 2011; Revised 17 November 2011; Accepted 27 November 2011

Academic Editor: Francesca Civano

Copyright (C) 2012 N. Neumayer and C. J. Walcher. This is an open access article distributed under the Creative Commons Attribution License, which permits unrestricted use, distribution, and reproduction in any medium, provided the original work is properly cited.

\begin{abstract}
We present new upper limits for black hole masses in extremely late type spiral galaxies. We confirm that this class of galaxies has black holes with masses less than $10^{6} M_{\odot}$, if any. We also derive new upper limits for nuclear star cluster masses in massive galaxies with previously determined black hole masses. We use the newly derived upper limits and a literature compilation to study the low mass end of the global-to-nucleus relations. We find the following. (1) The $M_{\mathrm{BH}}-\sigma$ relation cannot flatten at low masses, but may steepen. (2) The $M_{\mathrm{BH}}-M_{\text {bulge }}$ relation may well flatten in contrast. (3) The $M_{\mathrm{BH}}$-Sersic $n$ relation is able to account for the large scatter in black hole masses in low-mass disk galaxies. Outliers in the $M_{\mathrm{BH}}$-Sersic $n$ relation seem to be dwarf elliptical galaxies. When plotting $M_{\mathrm{BH}}$ versus $M_{\mathrm{NC}}$ we find three different regimes: (a) nuclear cluster dominated nuclei, (b) a transition region, and (c) black hole-dominated nuclei. This is consistent with the picture, in which black holes form inside nuclear clusters with a very low-mass fraction. They subsequently grow much faster than the nuclear cluster, destroying it when the ratio $M_{\mathrm{BH}} / M_{\mathrm{NC}}$ grows above 100. Nuclear star clusters may thus be the precursors of massive black holes in galaxy nuclei.
\end{abstract}

\section{Introduction}

Supermassive black holes (BHs) are thought to be ubiquitous in the nuclei of massive galaxies. The discovery of a number of tight correlations between the global properties of galaxies and the properties of their nuclei (e.g., [1-3]) has led astronomers to realize that the evolution of galaxies may be closely linked to their nuclear properties. However, the nuclei of galaxies do not only host massive BHs but also host massive star clusters, commonly called nuclear star clusters (NCs). (Note that we here make the distinction between nucleus, that is, the location at the very center, and nuclear star cluster. Often the NC has been called nucleus or stellar nucleus in the past, but this seems ambiguous to us).

The overall nucleation frequency is around $75 \%$ over all Hubble types ([4-6], hereafter B02), but NCs seem to be absent in the most massive galaxies [5,7]. NCs typically have stellar velocity dispersions of $15-35 \mathrm{kms}^{-1}$, effective radii of a few parsecs, and dynamical masses of $\sim 10^{6}-10^{7} M_{\odot}$ (B02 [8, 9]). Moreover, they show stellar populations of multiple ages [10-12], pointing towards them having a complex formation history. This might be related to their special location in the galaxy, as on average, NCs appear to sit at the photometric centre of their host galaxy $[6,13]$. We recently showed that for bulgeless galaxies their location also coincides with the kinematic centre, that is, the bottom of the potential well [14].

Intriguingly, NCs in late-type spirals and dwarf ellipticals follow relationships with their host galaxies that mirror the $M_{\mathrm{BH}}-\sigma$ and $M_{\mathrm{BH}}-M_{\text {bulge }}$ relationships of high-mass galaxies $[10,15,16]$, suggesting the possibility that the fueling and growth of $\mathrm{NCs}$ and $\mathrm{BH}$ are determined by similar processes, and that $\mathrm{BHs}$ and $\mathrm{NCs}$ should be grouped together into "central massive objects" (CMOs, [15]). The NC would then be nothing else than the failed $\mathrm{BH}$ [17]. In this picture, $\mathrm{BH}$ would form in high-density clumps typically located in highmass galaxies, while NCs form from lower-density clumps in lower-density disks. Recent simulation studies (e.g., [18, 19]) have been able to reproduce the formation of BHs through direct collapse models. If the collapse is quick-compared to the cooling time of the gas-a $\mathrm{BH}$ will form. If, however, the gas has sufficient time to cool and form stars, it will 
form an NC (see also the recent review in [20]). Competing formation scenarios for NCs are, however, equally successful. For example, recent work by Hartmann et al. [21] has shown that the observed properties of NCs are well reproduced by combining mergers of star clusters with the accretion of gas at a much later time in the history of a galaxy.

A further reason for interest in NCs and their BHs is that a number of authors [22-26] have found that dense clusters of young, massive stars can experience runaway coalescence of their most massive stars, leading to an intermediate mass black hole (IMBH, but see also [27]). It would then be tempting to identify NCs with the long-sought seeds for $\mathrm{BH}$ formation. An observational result supporting this view is that NCs and BHs can coincide [28, 29]; this is especially well studied in our own Galaxy $[30,31]$. On the other hand, parameter studies of the runaway collapse scenario (e.g., [32]) show that NCs are actually not in a region of parameter space that would be favourable to the collapse.

Of the many global-to-nucleus relations, the three most frequently referred to ones seem to be the $M_{\mathrm{BH}}-\sigma$ relation $[1,2]$, the $M_{\mathrm{BH}}-M_{\text {bulge }}$ relation [3], and the $M_{\mathrm{BH}}-n_{\text {Sersic }}$ relation [33]. As all these relations have been initially set up for the range of massive galaxies (i.e., $M_{\mathrm{BH}}>10^{8} M_{\odot}$ ), the low-mass range of BHs is not very well populated and holds most potential to find out which one of the three is more fundamental. A particularly interesting case is BHs and NCs in bulgeless galaxies. Indeed, while according to the $M_{\mathrm{BH}}-\sigma$ relation one would expect late-type, bulgeless spirals to host $\mathrm{BH}$ of mass $\leq 10^{6} M_{\odot}$, the $M_{\mathrm{BH}}-M_{\text {bulge }}$ relation is no longer "defined" for bulgeless galaxies, as the lack of a bulge would imply the absence of a black hole. On the other hand, exploring the low-mass end of the scaling relations, Green et al. [34] have derived reliable $\mathrm{BH}$ masses in spiral galaxies (with bulges) from maser measurements and find that these fall below the $M_{\mathrm{BH}}-\sigma$ relation of elliptical galaxies but seem consistent with the $M_{\mathrm{BH}}-M_{\text {bulge }}$ relation.

In fact both NCs and BHs have been found in bulgeless galaxies. For NCs see B02; for BHs see, for example, the cases of NGC4395 [28, 35], NGC1042 [36], NGC3621 [37, 38], and probably many more (see, e.g., [39-42]). On the other hand, very tight upper limits for the $\mathrm{BH}$ mass exist for some galaxies such as M33 [43, 44], but direct observational constraints are scarce because such small BHs are extremely difficult targets for dynamical searches and therefore very few objects have useful measurements. While it would thus seem tempting to declare that NCs are the central spheroids in bulgeless galaxies, this could lead to a paradox. Indeed, NCs have largely been identified with CMOs in massive galaxies, on the ground that they follow similar scaling relations as BHs. Identifying the same objects with the spheroid in lowmass galaxies would imply a transition in physical properties of the NC. Many observational hints seem to point against this possibility [9], the most important being that NCs have constant radius over Hubble type. A backdoor might be that Erwin and Gadotti [45] find that $\mathrm{BH}$ mass correlates with bulge mass (and no correlation with disk mass exists, [46]), while NC mass correlates better with total galaxy mass.

To conclude this introduction, measurements of the demographics of the lowest-mass $\mathrm{BHs}$ are an important goal. Their mass distribution encodes a fossil record of the mass scale and formation efficiency of the initial $\mathrm{BH}$ seeds at high redshift (e.g., [47]) and they hold the power to help us distinguish between different scenarios explaining the observed global-to-nucleus relations [48-52]. In order to increase the statistics in this particularly interesting lowmass regime, we have calculated $M_{\mathrm{BH}}$ upper limits for a sample of $9 \mathrm{NCs}$, for which integrated velocity dispersions had been published previously ([9], hereafter W05). We have also placed upper limits on $M_{\mathrm{NC}}$ for a sample of 11 galaxies with measured black hole masses. We have then used these upper limits in conjunction with a literature compilation to gauge which of the different proposed global-to-nucleus relations seem to hold best at the low-mass end.

\section{New Upper Limits for BHs in NCs}

2.1. Data. Our sample consists of 9 NCs culled from the HST/WFPC2 snapshot survey of B02. Imaging in the F804W filter is available from B02 and we refer to this paper for all details. All 9 NCs are resolved, even if some only barely. We here use the images as available through MAST to yield the surface brightness profile through a multi-Gaussian expansion (see the following).

VLT/UVES spectra with high $\mathrm{S} / \mathrm{N}$ and high spectral resolution have been obtained by W05. We use their velocity dispersion measurement. The properties of our sample are summarized in Table 1.

The sample selection for spectroscopic follow-up technically implied a slight bias to the more luminous among the NCs. Nevertheless, we expect this sample to be a fair representation of NCs in pure disk galaxies in general, as it covers the upper $2 / 3$ of the luminosity range of NCs.

2.2. Analysis. We constructed a dynamical model to estimate the mass and $M / L$ of the NCs and to put meaningful upper limits on the possible central black holes inside them. The first step in this process is developing a model for the light distribution. To parametrise the surface brightness profiles of the NCs and to deproject the surface brightness into three dimensions, we adopted a Multi-Gaussian Expansion (MGE; [53]). The MGE fit was performed with the method and software of Cappellari [54], on the HST I-band images deconvolved from the PSF (using a Tiny Tim PSF [55]). As most of the clusters are barely resolved in the HST images and shape measurements are therefore impossible, we assume spherical symmetry. Note that although the NCs in NGC300 and NGC7793 (the best resolved) are indeed round, this may be due to their host disks being seen face-on. Seth et al. [11] find that edge-on NCs can have quite disky outer isophotes.

We use the Jeans Anisotropic MGE (JAM) software by Cappellari [56] which implements the solution of the Jeans equations allowing for orbital anisotropy. The model has three free parameters: (i) the anisotropy, (ii) the mass of a central black hole $M_{\mathrm{BH}}$, and (iii) the $I$-band total dynamical $M / L$. From the velocity dispersion profile computed by JAM, we compute the luminosity-weighted velocity dispersion $\left(\sigma_{\mathrm{LW}}\right)$ over an aperture of 1 square arcsecond. This corresponds to the width of the UVES slit on the sky. We 
TABle 1: Properties of the sample of NCs in bulgeless galaxies.

\begin{tabular}{|c|c|c|c|c|c|c|}
\hline Galaxy & Type & $\mathrm{NC} r_{e}(\mathrm{pc})$ & $\sigma(\mathrm{km} / \mathrm{s})$ & $M / L^{\min }\left(M / L_{I, \odot}\right)$ & $M_{\mathrm{BH}}^{\max }\left(M_{\odot}\right)$ & $M_{\mathrm{BH}}^{\text {best }}\left(M_{\odot}\right)$ \\
\hline NGC 300 & SAd & 2.9 & $13 \pm 2$ & 0.41 & $1 \times 10^{5}$ & $1 \times 10^{2}$ \\
\hline NGC 428 & $\mathrm{SABm}$ & 3.36 & $24.4 \pm 4$ & 0.41 & $7 \times 10^{4}$ & $3 \times 10^{4}$ \\
\hline NGC 1042 & SABcd & 1.94 & $32 \pm 5$ & 0.07 & $3 \times 10^{6}$ & $2.5 \times 10^{4}$ \\
\hline NGC 1493 & SBcd & 2.6 & $25 \pm 4$ & 0.07 & $8 \times 10^{5}$ & $2.5 \times 10^{5}$ \\
\hline NGC 2139 & SABcd & 10.3 & $17 \pm 3$ & 0.02 & $4 \times 10^{5}$ & $1.5 \times 10^{5}$ \\
\hline NGC 3423 & SAcd & 4.18 & $30 \pm 5$ & 0.87 & $7 \times 10^{5}$ & $1.5 \times 10^{5}$ \\
\hline NGC 7418 & SABcd & 12.3 & $34 \pm 5$ & 0.10 & $9 \times 10^{6}$ & $1.5 \times 10^{5}$ \\
\hline NGC 7424 & SABcd & 7.4 & $16 \pm 2$ & 0.10 & $4 \times 10^{5}$ & $1.5 \times 10^{5}$ \\
\hline NGC 7793 & SAd & 7.7 & $25 \pm 4$ & 0.15 & $8 \times 10^{5}$ & $5 \times 10^{3}$ \\
\hline
\end{tabular}

iterate the computation of $\sigma_{\mathrm{LW}}$ over a grid of values for $M / L$ and $M_{\mathrm{BH}}$. The results are shown in Figure 1 which is directly comparable to Figure 8 of Barth et al. [37]. Direct comparison with the mass-to-light ratios obtained by W05 (thin solid vertical line) shows that the ratios scatter around 1.0, with no obvious systematic outliers. The small differences in the result can be attributed to the way in which the surface brightness was modelled (Multi-Gaussian expansion here versus direct deprojection in W05).

The maximum allowed mass of the black hole will be obtained when a minimum of mass is present in the form of stars. From Figure 1 one can easily read what $\mathrm{BH}$ mass would result if we assumed $M / L=0$ for the stars in the cluster. A more interesting lower limit to the $M / L$ comes from the spectral fitting with stellar population models. We exploit the fact that the age obtained by fitting a simple single stellar population to a composite stellar population is strongly biased to the age of the youngest population in that object which contributes significantly to the total luminosity (see, e.g., W06, [57]). The relevant values are tabulated in W06 and repeated in Table 1 along with the values derived as upper limits to the mass of a putative $\mathrm{BH}$ from the intersection between both thick solid lines in Figure 1. This is a conservative estimate for the $M_{\mathrm{BH}}$ upper limit. A more realistic value for $M_{\mathrm{BH}}$ can be derived from the intersection or asymptotic point of the model (thick solid curved line) with the best-fit $M / L$ from W05 (thin solid vertical line) in Figure 1. The resulting best-fit $M_{\mathrm{BH}}$ values are listed in Table 1.

We explicitly test the effect of velocity anisotropy on the modeling results and found very little change in the resultscertainly below our systematic uncertainties due to the lower limit to the mass-to-light ratio that we apply (see also [37]). We therefore neglect this effect for the rest of this paper.

\section{Global-to-Nucleus Relations}

We now plot the upper limits we have derived into figures showing existing correlations from earlier work. In these figures we typically have a comparison sample which is taken from a larger statistical study and we add a number of objects at the low-mass end from different sources in the literature. We have tried to be complete at the very lowest mass end of the relations. Further literature does exist, but typically, the $\mathrm{BH}$ masses exceed values of $\sim 10^{6} \mathrm{M}_{\odot}$ and the galaxies structural parameters have not been studied individually.

3.1. $M_{B H^{-}}-\sigma$ Relation. For Figure 2 , the $M_{\mathrm{BH}^{-}}-\sigma$ relation, the comparison sample, and relation are as compiled by Gültekin et al. ([58], black open symbols). We extend this compilation with recent maser measurements by Greene et al. ([34], green crosses). Active AGNs are denoted by blue stars; these are NGC4395 [28, 59] and POX52 [60]. In principle, NGC1042 from the present work falls also into this category (see, [36]) but is plotted as a filled red circle. Previous upper limits for nonactive nucleated galaxies are plotted as open blue circles: M33 [43, 44], NGC205 [61], IC342 [62], and NGC404 [63]. We also plot the globular clusters G1 [64], $\omega$ Cen $[51,65,66]$, and NGC6388 [67] as green open circles. The verdict on the usefulness of these measurements is still out, with strong contrasting claims by other authors that there is no evidence for a black hole in $\omega$ Cen $[68,69]$ and in G1 (e.g., [70]). We nevertheless use the derived values in a spirit of adventure, that is, what would it mean if these measurements were correct? Finally, the new upper limits derived in this work are denoted by filled red circles. It emerges that a flattening of the relation is not consistent with the current measurements. It may well be that a downwards bending would be necessary, if more stringent upper limits such as that for M33 would be published.

3.2. $M_{B H}-M_{\text {bulge }}$ Relation. For Figure 3 , the $M_{\mathrm{BH}}-M_{\text {bulge }}$ relation, the comparison relation and sample are taken from Häring and Rix [3] (filled black circles), while the other data points come from the same sources as in Figure 2. There is a hint towards a flattening of the relation with the lowest spheroid masses, but it will be difficult to confirm this without much better estimates of the masses of IMBHs. On the other hand a steepening, that is, bulges that are too massive for their BHs, has been mentioned by Greene et al. $[34,71]$. If there are $\mathrm{BHs}$ in galaxies with no bulges as well as bulges that are too massive for their $\mathrm{BHs}$, it seems clear that the $M_{\mathrm{BH}}-M_{\text {bulge }}$ relation must suffer from large scatter at small masses.

3.3. $M_{B H^{-}} n_{\text {Sersic }}$ Relation. For Figure 4 , the $M_{\mathrm{BH}}-n_{\text {Sersic }}$ relation, the comparison relation, and sample are taken from 


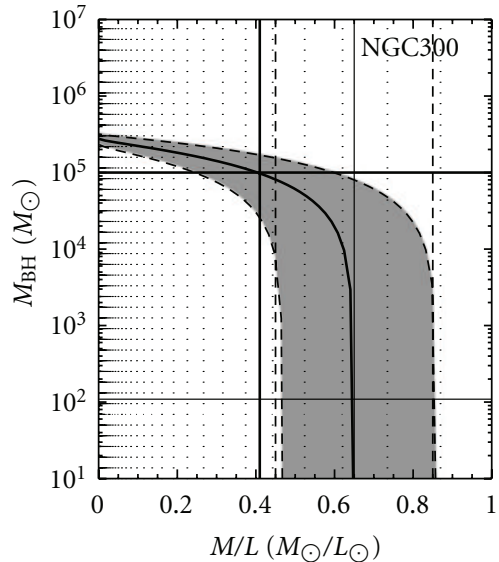

(a)

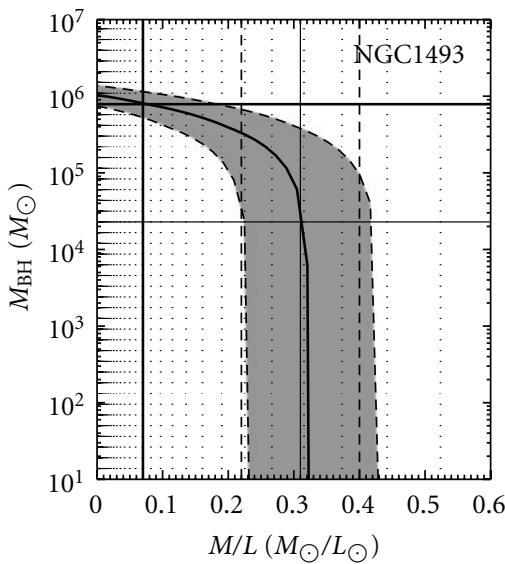

(d)

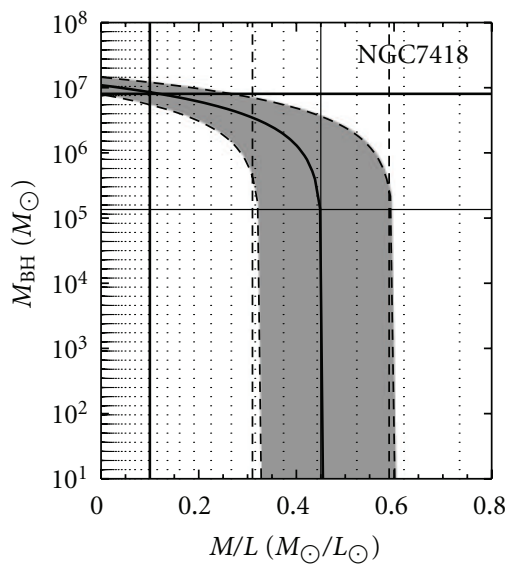

(g)

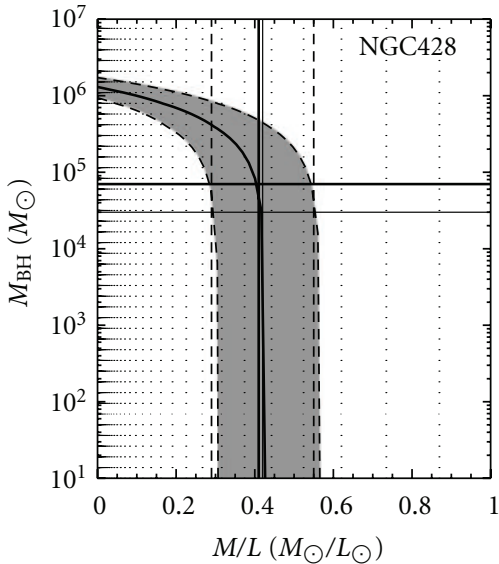

(b)

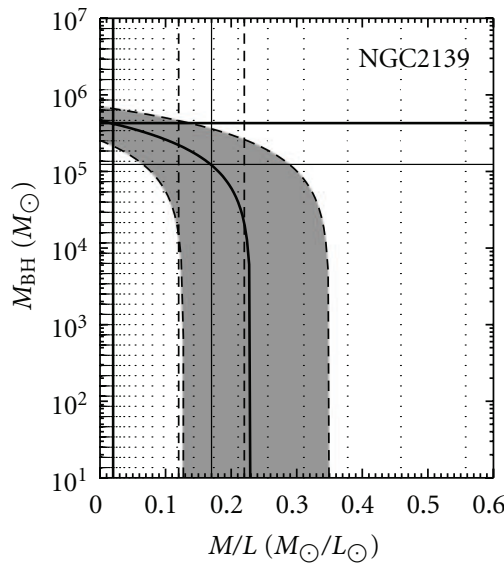

(e)

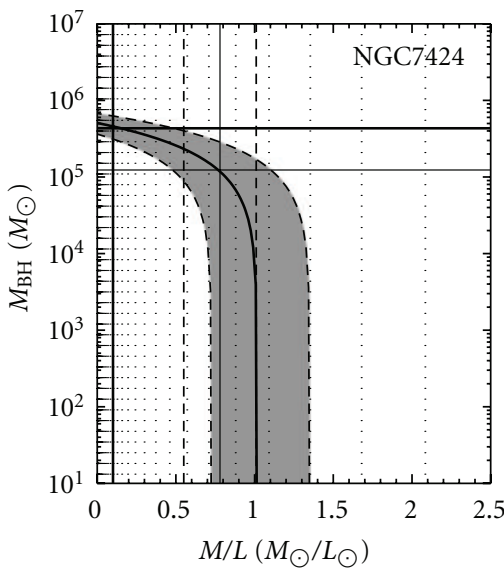

(h)

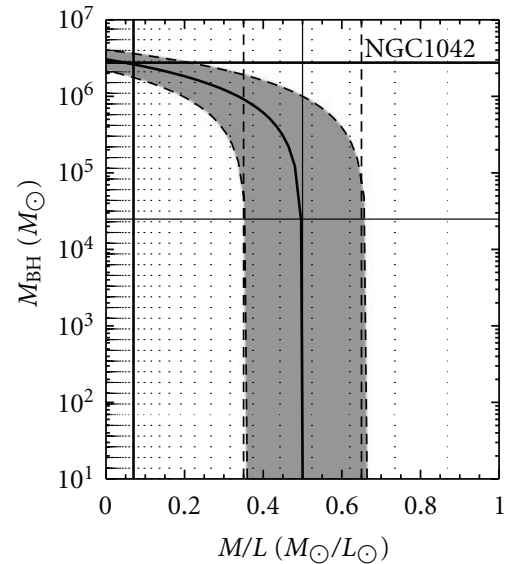

(c)

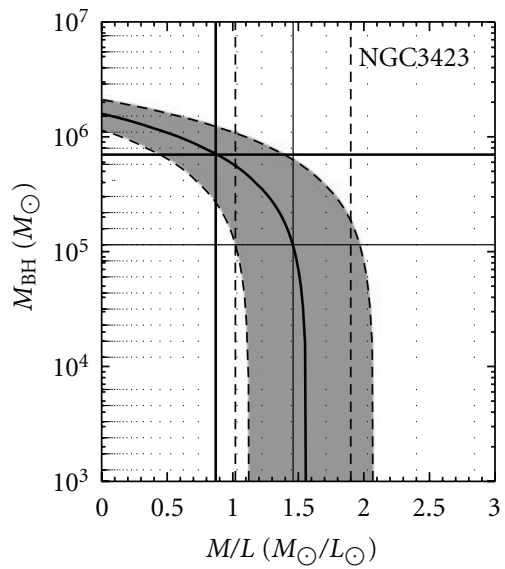

(f)

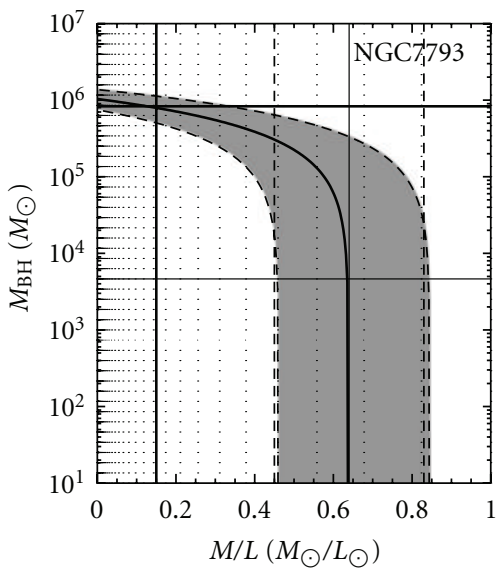

(i)

Figure 1: The $M / L$ ratio assumed for the stellar population against the mass of the putative black hole for each of the nine nuclear clusters. Models falling onto the right solid vertical line have the same velocity dispersion as given in Table 1 for each cluster (dashed lines are upper and lower uncertainties). We also draw a vertical, full line (left) for the minimum mass-to-light ratio compatible with the observed spectrum of the stellar population in the cluster. The horizontal solid lines indicate the black hole masses referring to the two different $M / L$ values quoted previously. For the minimum $M / L$ we find a firm upper limit to the black hole mass $M_{\mathrm{BH}}^{\max }$ (upper line) and for the best-fit stellar populations $M / L$ we get $M_{\mathrm{BH}}^{\text {best }}$ (lower line). 


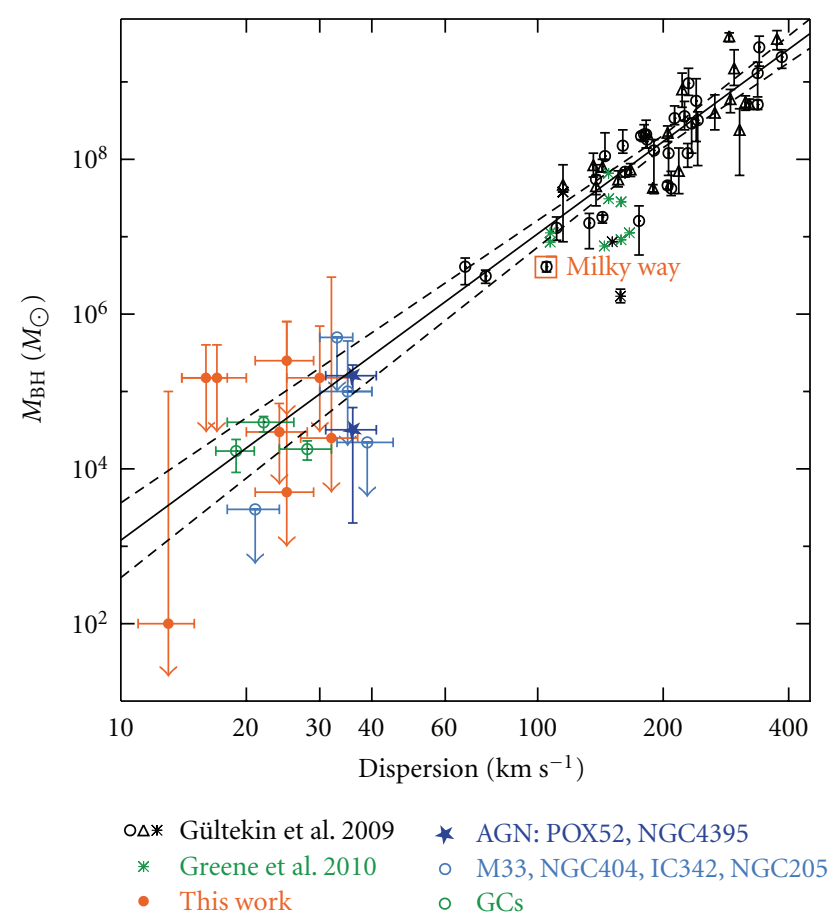

Figure 2: The relation between the mass of the $\mathrm{BH}$ and the velocity dispersion of the spheroid around it. We plot the objects as listed in the text. The lines give the best fit of Gültekin et al. [58].

Graham and Driver [33] (filled black circles), while the other data points come from the same sources as in Figure 2. We have also assembled measurements of the Sersic $n$ from literature sources for all objects with published $\mathrm{BH}$ masses. For the galaxies with $M_{\mathrm{BH}}$ limits newly derived in the present paper, Sersic $n$ was derived from the following literature sources: Ganda et al. [72] for NGC1042 and NGC3423 and Weinzirl et al. [73] for NGC2139. For NGC300 and NGC428 Spitzer IRAC $3.6 \mu \mathrm{m}$ images were downloaded from the Spitzer Heritage Archive (http://sha.ipac.caltech.edu/) and Sersic $n$ was determined using the GALFIT software [74]. For NGC1493, NGC7424, and NGC7793 the corresponding images were obtained from the 2MASS archive (http://irsa.ipac.caltech.edu/) and again fitted with GALFIT. All galaxies were fit using one PSF component for the central NC, one Sersic component representing the disk, and one constant sky component. All parameters were left free to be fit for. The webpages provide appropriate point spread functions; although all of our targets are nearby and therefore well resolved, the resulting Sersic $n$ is almost independent of the PSF used in GALFIT. We caution that the resulting Sersic $n$ may depend heavily on the radial range used in the fitting. To cite two extreme examples, the Sersic $n$ of NGC300 is independent of the radial range used within $\Delta(n)=0.1$. On the other extreme, the Sersic $n$ for NGC1493 varies between $\sim 1.3$ and the reported value of $\sim 2.5$. It is beyond the scope of the current paper to derive a physically meaningful fit range that would put the physical meaning of the Sersic $n$ on firmer ground. We emphasize that it is despite the cited uncertainties that the relation between $M_{\mathrm{BH}}$ and Sersic $n$ seems to hold.

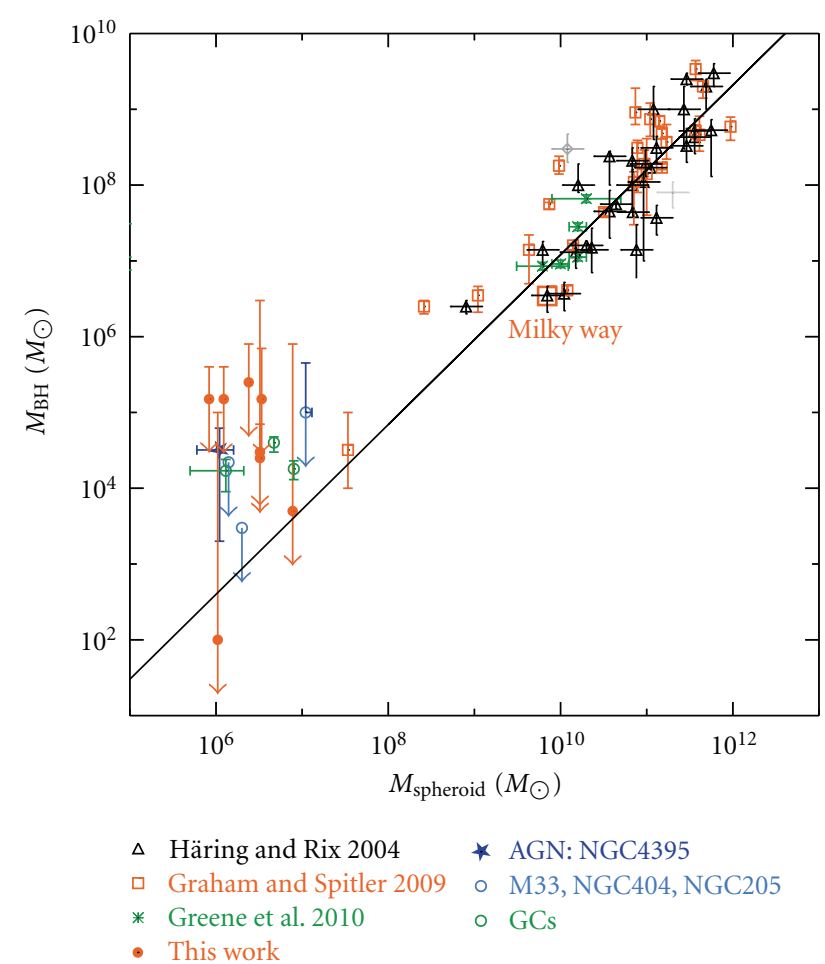

Figure 3: The BH mass versus the spheroid mass (bulge, GC, NC). We plot the objects as listed in the text. The line indicates the bestfitting relation of Häring and Rix [3].

Figure 4 shows two interesting features. (1) Because the relation fitted by Graham and Driver [33] curves down at $n=1$, a large range of $\mathrm{BH}$ masses is allowed in this regime, which clearly allows for the scatter that seems to emerge as a common trend in the previous two nucleus-to-global relations. (2) There are significant outliers in this plot, in the sense that some low-mass galaxies can have too high Sersic $n$ for their $\mathrm{BH}$ mass.

3.4. $M_{B H}-M_{N C}$ Relation. In Figure 5 we show the relation between $M_{\mathrm{BH}}$ and $M_{\mathrm{NC}}$ (compare $[29,75]$ ). We have plotted objects already used above, for which determinations of both $M_{\mathrm{BH}}$ and $M_{\mathrm{NC}}$ exist. In searching for a high-mass comparison sample we have made use of the compilations by Graham and Spitler [75] and Gültekin et al. [76] from which we also take the distances. Where not available, we have then proceeded to derive upper limits to the NC masses either from the literature or from own fits to archival HST images. (Thorough work deriving consistent photometry and structural parameters for NCs across the entire Hubble sequence for large swaths of the HST archive is badly needed, but is beyond the scope of the current work. Note that one focus of such work could be the distinction (if any clear distinction exists) between NCs and nuclear disks. In the case of NGC4342, for example, the upper limit we give on the NC mass is not only observationally uncertain, but also conceptually uncertain. As Scorza and van den Bosch [77] discuss, a relation between the nuclear disk mass and the $\mathrm{BH}$ is as plausible as between the $\mathrm{NC}$ and the $\mathrm{BH}$. Indeed, some 


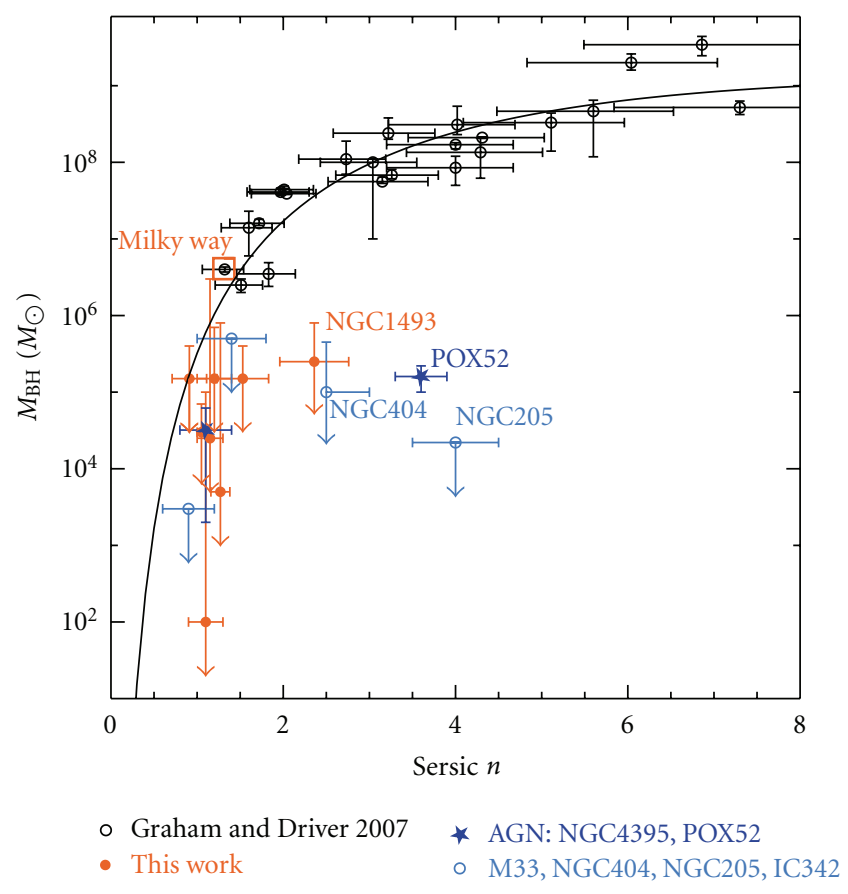

FIgUre 4: The mass of the BH against the Sersic index of the host bulge or disk. We plot the objects as listed in the text. The largest outliers are NGC205 (with $n=4), \operatorname{POX} 52(n=3.6)$, NGC404 $(n=$ 2.5), and NGC1493 $(n=2.4)$.

NCs may turn out to be nuclear discs on close inspection (compare [11])).

We now discuss the ways that we have obtained upper limits for the NC masses galaxy by galaxy. We strongly emphasize that we have tried to obtain upper limits to rather than real measurements of the NC mass. Real measurements of NC masses can only be carried out by a combination of dynamical modeling and spectral analysis to determine the relative influence of the AGN and possible varying $M / L$ ratios. We rather aim to be conservative with respect to all uncertainties affecting our estimates of upper limits to the NC masses. Our resulting upper limits are listed in Table 2. For the following 5 objects we estimated upper mass limits from the literature only.

NGC4486 (M87): the bright nucleus is dominated by AGN light. There is no evidence for an NC. We therefore use Figure 7 of Gebhardt and Thomas [78], which shows the enclosed stellar mass within the central arcsec to be $2 \times 10^{8} M_{\odot}$. This is consistent with an estimate from Young et al. [79], which gives a total of $M=5 \times 10^{9} M_{\odot}$ within a radius of $100 \mathrm{pc}$ and $M / L=60$, thus leading to an estimate of the stellar mass within that radius of $3 \times 10^{8} M_{\odot}$, assuming that the stellar $M / L=4$. We emphasize that this is the total stellar mass within a radius comparable to the radii of typical NCs and therefore gives an upper limit to $M_{\mathrm{NC}}$. We do not claim that M87 actually hosts a stable NC at its centre.

NGC4374 (M84): an AGN has been shown to exist by Bower et al. [80], with very weak stellar features. To estimate an upper mass to the NC in NGC4374 we use the paper by Walsh et al. [81], which yields a $\mathrm{BH}$ mass estimate of $4 \times 10^{8} M_{\odot}$. Their Figure 4 shows the circular velocity

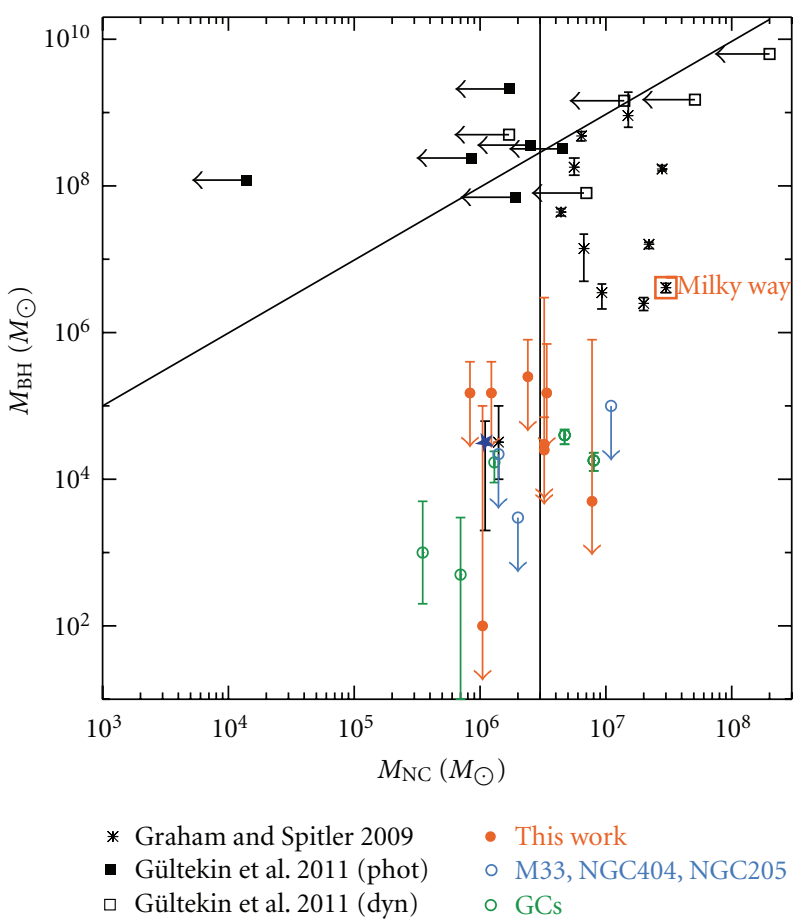

Figure 5: The mass of the BH mass versus the NC mass. We plot the objects as listed in the text. The two full lines indicate an NC mass of $3 \times 10^{6} M_{\odot}$ and a $M_{\mathrm{BH}} / M_{\mathrm{NC}}$ mass ratio of 100 . These lines separate $\mathrm{NC}$-dominated galaxy nuclei (lower left of both lines) from $\mathrm{BH}-$ dominated galaxy nuclei (upper left of both lines) and a transition region (to the right of both lines).

profiles due to the $\mathrm{BH}$ and the stellar mass, respectively. Assuming a distance to M84 of $17 \mathrm{Mpc}$ yields $70 \mathrm{pc} / \mathrm{arcsec}$. Assuming an NC radius of 10 and a stellar $M / L=4$, we obtain that at $10 \mathrm{pc}$ radius the circular velocity due to the $\mathrm{BH}$ is $400 \mathrm{~km} / \mathrm{s}$, while the circular velocity due to the remaining stellar mass is smaller than $50 \mathrm{~km} / \mathrm{s}$. To obtain an estimate of the upper limit for a putative NC, we need to correct for the different masses and for the different spatial distribution (point-like versus extended). From the virial theorem, we can scale the velocity quadratically. From Table 2 in the current work it can be seen that a conservative factor (i.e., one that gives a lot of stellar mass) for the conversion from pointlike to extended would be a factor of 10 . The upper limit for the stellar mass within 10 then becomes $M_{\mathrm{NC}} / M_{\mathrm{BH}}=$ $10 * 50^{2} / 400^{2}=0.15$, thus yielding an NC upper mass limit of $6.3 \times 10^{7} M_{\odot}$. Walsh et al. [81] also state that stellar mass is a negligible contributor to their mass budget; it is therefore entirely possible that no NC exists in that galaxy.

NGC4261: the central luminosity distribution is complex, with a nuclear disk and a luminous nuclear source which seems to be dominated by an AGN; at least a radio jet is present [82]. There is thus no clear evidence in favour of any NC. Ferrarese et al. [82] find that $M / L_{V}=2100$ within the inner $14.5 \mathrm{pc}$. A maximum $M / L_{V}$ for stellar populations is 7 . We thus obtain that $7 / 2100$ of the central mass within $15 \mathrm{pc}$ can be in stars, which is $5 \times 10^{8} / 300=1.6 \times 10^{6} \mathrm{M}_{\odot}$. 
TABLE 2: Sample of galaxies for which new properties were derived in this paper.

\begin{tabular}{|c|c|c|c|c|c|c|c|c|}
\hline Galaxy & Type & $\sigma(\mathrm{km} / \mathrm{s})$ & Dist $\left(M_{\mathrm{pc}}\right)$ & $M_{\mathrm{BH}}\left(M_{\odot}\right)$ & $M_{\mathrm{NC}}\left(M_{\odot}\right)$ & Sersic $n$ & $M_{\text {Bulge }}\left(M_{\odot}\right)$ & Ref \\
\hline group 1 & & & & $\times$ & & & & \\
\hline NGC 300 & SAd & $13 \pm 2$ & 2.2 & $<1 \times 10^{2}$ & $1 \times 10^{6}$ & 1.1 & - & {$[105]$} \\
\hline NGC 428 & $\mathrm{SABm}$ & $24.4 \pm 4$ & 16.1 & $<3 \times 10^{4}$ & $3 \times 10^{6}$ & 1.05 & - & [105] \\
\hline NGC 1042 & SABcd & $32 \pm 5$ & 18.2 & $<2.5 \times 10^{4}$ & $3 \times 10^{6}$ & 1.15 & - & [105] \\
\hline NGC 1493 & SBcd & $25 \pm 4$ & 11.4 & $<2.5 \times 10^{5}$ & $2 \times 10^{6}$ & 2.36 & - & {$[105]$} \\
\hline NGC 2139 & SABcd & $17 \pm 3$ & 23.6 & $<1.5 \times 10^{5}$ & $8 \times 10^{5}$ & 1.53 & - & {$[105]$} \\
\hline NGC 3423 & SAcd & $30 \pm 5$ & 14.6 & $<1.5 \times 10^{5}$ & $3 \times 10^{6}$ & 1.20 & - & {$[105]$} \\
\hline NGC 7418 & SABcd & $34 \pm 5$ & 18.4 & $<1.5 \times 10^{5}$ & $6 \times 10^{7}$ & - & - & {$[105]$} \\
\hline NGC 7424 & SABcd & $16 \pm 2$ & 10.9 & $<1.5 \times 10^{5}$ & $1 \times 10^{6}$ & 0.91 & - & {$[105]$} \\
\hline NGC 7793 & SAd & $25 \pm 4$ & 3.3 & $<5 \times 10^{3}$ & $8 \times 10^{6}$ & 1.27 & 一 & [105] \\
\hline group 2 & & & & & $\times$ & & & \\
\hline NGC 4486 & E1 & $375 \pm 18$ & 17.0 & $6.3 \times 10^{9}$ & $<2 \times 10^{8}$ & 6.86 & $6.0 \times 10^{11}$ & {$[68]$} \\
\hline NGC 4374 & E1 & $296 \pm 14$ & 17.0 & $1.5 \times 10^{9}$ & $<6.3 \times 10^{7}$ & 5.60 & $3.6 \times 10^{11}$ & [41] \\
\hline NGC 1332 & So & $321 \pm 14$ & 19.6 & $1.45 \times 10^{9}$ & $<1.4 \times 10^{7}$ & - & - & {$[60]$} \\
\hline NGC 3031 & $\mathrm{Sb}$ & $143 \pm 7$ & 4.1 & $8 \times 10^{7}$ & $<7 \times 10^{6}$ & 3.26 & - & [37] \\
\hline NGC 4261 & E2 & $315 \pm 15$ & 33.4 & $5 \times 10^{8}$ & $<1.7 \times 10^{6}$ & 7.30 & $3.6 \times 10^{11}$ & [70] \\
\hline group 3 & & & & & $\times$ & & & \\
\hline NGC 4649 & E2 & $385 \pm 19$ & 16.5 & $2.1 \times 10^{9}$ & $<2 \times 10^{6}$ & 6.04 & $4.9 \times 10^{11}$ & {$[103]$} \\
\hline NGC 3998 & So & $305 \pm 15$ & 14.9 & $2.4 \times 10^{8}$ & $<8.5 \times 10^{5}$ & - & - & [98] \\
\hline NGC 2787 & SB0 & $189 \pm 9$ & 7.9 & $0.7 \times 10^{8}$ & $<1.9 \times 10^{6}$ & 1.97 & - & [95] \\
\hline NGC 3379 & E0 & $206 \pm 10$ & 11.7 & $1.2 \times 10^{8}$ & $<1.4 \times 10^{4}$ & 4.29 & $6.8 \times 10^{10}$ & [108] \\
\hline NGC 4342 & So & $225 \pm 11$ & 18.0 & $3.6 \times 10^{8}$ & $<2.5 \times 10^{6}$ & 5.11 & $1.2 \times 10^{10}$ & {$[13]$} \\
\hline NGC 4291 & E2 & $242 \pm 12$ & 25.0 & $3.2 \times 10^{8}$ & $<5 \times 10^{6}$ & 4.02 & $1.3 \times 10^{11}$ & [103] \\
\hline
\end{tabular}

Galaxies for group 1 are from W05, and we here derived upper limits on the black hole mass and Sersic $n$. Galaxies for groups 2 and 3 are from Gultekin et al. [76]. For group 2 objects we derived upper limits on the NC mass via dynamical arguments, while for group 3 objects we used photometry to derive $M_{N C}$ upper limits. For groups 2 and 3 Sersic $n$ values are taken from Graham and Driver [33], bulge masses are from Häring and Rix [3], and velocity dispersions are from Hyperleda. The newly derived quantities are marked with an $\times$ at the top of the respective column. References for black hole masses are. (1) this work, (2) Gebhardt and Thomas [78], Gebhardt et al. [109], (3) Bower et al. [110], (4) Rusli et al. [83], (5) Devereux et al. [84], (6) Ferrarese et al. [82], (7) Gebhardt et al. [111], (8) De Francesco et al. [88], (9) Sarzi et al. [112], (10) Gebhardt et al. [90]; Shapiro et al. [113], and (11) Cretton and van den Bosch [89].

NGC1332: there is no firm evidence for an NC, although the surface brightness profile of Rusli et al. [83] hints at a central luminosity excess within the central arcsec. The dynamical model of Rusli et al. [83] gives a central stellar luminosity density of $4 \times 10^{12} L_{\odot} \mathrm{kpc}^{-3}$. For an NC of $5 \mathrm{pc}$ radius this yields an NC luminosity of $2 \times 10^{6} L_{\odot}$ in the Rband. With $M / L_{R}=7$ (also according to [83]), $M_{\mathrm{NC}} \max$ is $1.4 \times 10^{7} M_{\odot}$.

NGC3031: Devereux et al. [84] list values of stellar mass within radius in their Table 3. From their Figure 3, it is clear that the nuclear source is not extended; there is thus no evidence for the presence of an NC. To estimate an upper mass limit for the NC, we assume an NC radius of $7 \mathrm{pc}$ (compare [5,6]); the upper limit to $M_{\mathrm{NC}}$ is then $7 \times 10^{6} M_{\odot}$.

For the following 6 objects no NC mass estimate was available. We therefore turned to the HST images as downloaded from the Hubble Legacy Archive. We have then used GALFIT [74] to derive the magnitudes of the NCs. Because all NCs we treat in this last step are in early-type galaxies, we can assume that their ages range between 1 and $10 \mathrm{Gyr}$, yielding an estimate of the allowed range for the $M / L$ ratio. For most cases we used the F814W filter on either ACS or WFPC2, setting the allowed range of $M / L$ between 1 and
4. Much more sophisticated modeling of the photometry, while possible, would yield only marginally better estimates of the total stellar mass of the NC for several reasons. (1) The star formation histories (SFHs) of NCs are unknown and, indeed, likely to be semirandom, repetitive bursts of star formation. Therefore no strong prior can be applied to the $\mathrm{SFH}$. Because the oldest stellar populations are the faintest per unit mass, the resulting uncertainty on $M / L$ is of order factor 2. (2) The photometry of the NCs is in itself uncertain. We have made use of realistic PSFs from either Jee et al. [85] (http://acs.pha.jhu.edu/ mkjee/acs_psf/) or from Tiny Tim (http://www.stsci.edu/hst/observatory/focus/TinyTim). It is much less certain what the ideal profile for the surface brightness of the host galaxy should be though (compare [86, 87]). We have used one single Sersic, as we are only interested in subtracting the host, not in describing it. Nevertheless, we estimate that the use of different profiles (2 Sersics, Nuker, etc.) could impact the total photometry of the NC by up to 0.5 or even 1 magnitude (compare, e.g., the central extrapolations of [6]). We therefore have chosen to let these uncertainties be reflected in the errorbars of the NC mass estimate, rather than trying to hide them somewhere within a sophisticated analysis. 
NGC4649: no nuclear source is visible (as also found by [75]). We first fit this galaxy with a single Sersic. When additionally forcing in a point source (GALFIT PSF component) of different magnitudes (20, 20.5, 21,21.5, 22), the resulting oversubtraction can be seen clearly in the residual image for as faint as $m_{I}=21.0$. We use this value as a conservative upper limit to the NC magnitude. This results in an upper mass limit of $2 \times 10^{6} M_{\odot}$.

NGC4291: we attempted the same procedure as before. However, due to a flat central surface brightness profile, our simple Sersic fit by itself produced an oversubtraction of the central flux, not allowing us to use the exact same procedure as for NGC4649. Nevertheless, the HST image clearly shows the absence of any point source in the center. We therefore assumed the same limit as before, that is, $21 \mathrm{mag}$ in F814W, which results in $M_{\mathrm{NC}}=5 \times 10^{6} M_{\odot}$.

NGC3998: after the GALFIT fit, a clear spiral structure and a bar are seen in the residuals. The central light source was modeled as a Sersic with an effective radius of $0.2^{\prime \prime}$ and a Sersic $n=0.1$, making us believe it is unresolved. De Francesco et al. [88] classify this galaxy as a LINER; thus the central source is AGN-light dominated. Therefore our photometrically derived NC mass of $8.5 \times 10^{5} M_{\odot}$ again is a conservative upper limit.

NGC4342: the fit with GALFIT was difficult, with 4 Sersic components in the final fit. The final solution was chosen to oversubtract the NC. Again we have a conservative upper limit of $21.85 \mathrm{mag}$ corresponding to $M_{\mathrm{NC}}=2.5 \times 10^{6} \mathrm{M}_{\odot}$, using an $M / L$ of 6.5 in I from Cretton and van den Bosch [89]. Contamination from AGN light is also possible, making our upper mass limit more robust.

NGC3379 (M105): the NC is visible in Gebhardt et al. [90], but not mentioned there. Graham and Spitler [75] note this galaxy as unnucleated. Two extended components with Sersic $n \sim 1$ and one very compact source with Sersic $n \approx 1 / 2$ (i.e., Gaussian surface brightness profile) and $r_{e}=$ $0.2^{\prime \prime}$ give a good fit to this object. The measured integrated magnitude of the central point source is 25.7 in the F814W of WFPC2, corresponding to $M_{\mathrm{NC}}=1.4 \times 10^{4} M_{\odot}$. We used $M / L_{F} 814 W=3$ as a suitable upper limit to the $M / L$.

NGC2787: this galaxy was analyzed in Peng et al. [74] and the nuclear photometry is taken from that source. We used $M / L_{F} 547 M=3$ as a suitable upper limit to the $M / L$. Thus, the NC upper mass limit is $1.9 \times 10^{6} \mathrm{M}_{\odot}$.

Note that in the galaxies NGC4486, NGC4374, and NGC3379 a luminous nuclear source is clearly seen. While this could all be AGN light, we see no way to ascertain the absence of an NC. In contrast to Graham and Spitler [75] we only claim to be able to derive an upper limit to the $\mathrm{NC}$ mass, rather than excluding an NC all together. Note also that a stellar cusp containing $10 \%$ of the $\mathrm{BH}$ mass is predicted around any $\mathrm{BH}[91]$.

\section{Discussion}

We now discuss and interpret a number of features we saw in the previous section, with the aim to discuss ideas that emerge from these figures but, to our knowledge, have not been discussed in the literature before. The ultimate aim of our study is of course to contribute to a consistent physical picture of black hole and nuclear cluster growth.

In Figure 4, one relation between the global galaxy properties and $M_{\mathrm{BH}}$ holds for a large range of $n$, independent of the presence of a bulge. Outliers are rather low-mass galaxies (and not low-mass black holes).

We stress that this relation is purely observational at this stage. Due to the heterogeneous assembly of the Sersic $n$ values (literature, own fitting) the physical region represented by them is not always the same. In particular some of the galaxies do not contain a bulge, while for others the Sersic $n$ has been explicitly measured for the bulge component. The existence of a relation seems evidence that indeed the measurement of Sersic $n$ is meaningful. In particular, no conspiracy is obvious that would fundamentally bias our measurements in the sense of producing a spurious correlation. It thus seems to us that even independently of the exact details of the derivation of the Sersic $n$, it clearly describes a property of the galaxy that is relevant for the $\mathrm{BH}$ mass. A question to ask is then whether we fully understand the physical implications of that relation, and whether we could potentially reduce the relation to underlying intrinsic distribution of galaxy properties (e.g., if Sersic $n$ was related to bulge mass in a very tight manner, we might be tempted to argue that bulge mass is the more fundamental measurement). We believe that the present paper cannot resolve this question but hope it provides motivation to explore these issues further.

To venture a possible physical interpretation of the outliers from the relation we note the following: it could be that the transformation process from disk galaxy to spheroid is different in this galaxy mass regime. While BHs in massive galaxies grow during the morphological transformation process of their host galaxies, $\mathrm{BH}$ s in low-mass galaxies are not affected (fed) during the transformation process. It might be worthwhile exploring through simulations, whether this has to do with a possible transformation dichotomy, that is, mergers versus harassment. It is worth pointing out here that such a dichotomy does not seem to be immediately apparent from the age or metallicity profiles, as these seem not to depend on mass [92].

Figure 5 has not been published previously in this form to the best of our knowledge (though compare $[29,75]$ for similar representations) and may yield considerable insight on the relation between $\mathrm{NCs}$ and BHs. An immediate conclusion from this figure is that $\mathrm{BH}$ and their host NCs do not share the same intimate connection as BHs and their host spheroids. It rather seems that in galaxies with a high total mass, or alternatively a sizable spheroid, the $\mathrm{BH}$ has been able to grow independently of the NC, thus being able to reach comparable masses. In galaxies or star clusters unaffected by spheroid growth, as, for example, the GCs, M33, and others, it seems the $\mathrm{BH}$, if existent, is only a very small portion of the mass of the NC.

Figure 5 (and similar figures, see $[7,29,75]$ is still in an early phase and we believe that further studies in the field will attempt to fill in the high- and low-mass end of the $\mathrm{BH}$ mass regime with more $\mathrm{NC}$ masses and $\mathrm{BH}$ masses, respectively. Nevertheless it seems that two extreme ends can be identified, 
with a transition region in between. At the low $\mathrm{BH}$-mass end, there is little evidence for the presence of any $\mathrm{BH}$; yet NCs seem to be common (B02). On the other hand no nuclear $\mathrm{BH}$ has been found that is not surrounded by an $\mathrm{NC}$ in this regime. If GCs with $\mathrm{BHs}$ are indeed the remnants of accreted satellite galaxies (e.g., [93-96]) and if indeed they lie on the $M_{\mathrm{BH}}-\sigma$ relation, this would imply, however, that at this stage $\mathrm{BH}$ growth is linked to $\mathrm{NC}$ growth much in the same way that $\mathrm{BH}$ growth is later tied to galaxy growth. A close look suggests indeed that some physical processes that occur in or with NCs, such as gas accretion [97-99] and merging [100-105], are quite similar to those experienced by galaxies. An alternative to the assumption that the process giving rise to the $M_{\mathrm{BH}}-\sigma$ is so astoundingly generic is of course that the BH mass measurements in GCs may be subject to the "expectation bias", that is, when the measurement is in accordance with the expectations of the community they get accepted more easily.

At the very high-mass end of the $\mathrm{BH}$-mass range, the $\mathrm{BH}$ is much more massive than the NC. On the other hand, this is the region where global-to-nucleus relations hold best. This could happen through two mechanisms: (1) either the galaxies in question never had a sizable NC, possibly because their central BHs grew early on in the age of the universe, thus stopping NC growth [106], or (2) massive BHs destroy their host NCs. Figure 5 in its current form suggests that this may happen at a mass ratio of $\geq 100$ or alternatively when the $\mathrm{BH}$ radius of influence is of the same size as the NC radius. Loss cone depletion and core formation in early-type galaxies are well-studied mechanisms, that would amply suffice to destroy the preexisting NC [107].

Bekki and Graham [108] have examined an alternative solution for the disappearance of NCs for massive galaxies. Their argument relies on the mergers that are responsible for the morphological transformation from disk-dominated to bulge-dominated galaxies. They show that NCs can be significantly heated and thus be made susceptible to destruction during the merger event. The picture painted here differs significantly from that painted in Bekki and Graham [108] in that we put weight on the importance of the $\mathrm{BH}$ for destroying the NC. Indeed, what determines NC disappearance does not seem to be galaxy morphology, as most early-type galaxies have NCs. Rather, there is evidence for an upper limit to the $\mathrm{BH} / \mathrm{NC}$ mass ratio, arguing strongly for a pivotal role of this ratio in leading to NC disruption.

The intermediate mass or transition regime may possibly lie between two boundaries, that is, above NC masses of $5 \times 10^{6} M_{\odot}$ and below a $M_{\mathrm{BH}} / M_{\mathrm{NC}}$ mass ratio of 100 . In this intermediate mass regime, while $\mathrm{BHs}$ have grown by at least 2 orders of magnitude, and probably more than 4 as compared to the NC-dominated regime, the NC grows by at most a factor of 10. There thus is some common growth; yet it does not occur in parallel. On the other hand, this is the region of most scatter in the typical global-to-nucleus relations. This phase would thus be characterized as a transition phase between NC-dominated nuclei and $\mathrm{BH}$-dominated nuclei.

Does Figure 5 imply that NCs do not grow by the same processes as their $\mathrm{BHs}$ and is this a serious setback to the grouping together of NCs and BHs into CMOs [15]?
That NCs and BHs need not grow in parallel has been emphasized by Nayakshin et al. [106], where both types of objects rather grow in competition for the same gas reservoir. Nayakshin et al. [106] ask whether the $\mathrm{BH}$ can prevent the NC from growing through its feedback and postulate that this is the case when the gas accretion rate is smaller than the Eddington rate. This picture is attractive in explaining Figure 5 because it naturally explains the three regimes$\mathrm{NC}$ dominated, $\mathrm{NC} / \mathrm{BH}$ transition, and $\mathrm{BH}$ dominated. Nevertheless, given the very low accretion rates observed in bulgeless galaxies and the presence of significant $\mathrm{BHs}$ in at least a few of them, this picture seems to break down exactly for the NC-dominated regime.

Discrimination between the different scenarios envisaged in the literature seems to be mostly an observational question at present. At low masses the error bars on $\mathrm{BH}$ measurements are typically very large, while NC masses are well measured. At high masses, $\mathrm{BH}$ masses are more accurate while the uncertainties for NC masses increase, due to resolution problems of the NCs above the underlying galaxies. We need both reliable $\mathrm{BH}$ and $\mathrm{NC}$ masses to see what the exact locus of points in this plot is. If there is a smooth transition, making the sequence look like a closed parenthesis, this would imply that the destruction of the NC due to the growing black hole is a slow process. If there really is a well-defined transition at $M_{\mathrm{BH}} / M_{\mathrm{NC}}=100$, then this would imply either that the process of NC destruction is very fast or that these galaxies never had an NC.

\section{Conclusions}

We have computed new upper limits for the masses of intermediate mass black holes in 9 pure disk galaxies with very low $\mathrm{BH}$ masses. We also computed upper limits to the masses of nuclear star clusters in the nuclei of galaxies with previously determined massive BHs. We plot these upper limits on the three global-to-nucleus relations $M_{\mathrm{BH}}-\sigma$, $M_{\mathrm{BH}}-M_{\text {bulge }}$, and $M_{\mathrm{BH}}-$ Sersic $n$, as well as on a new figure that compares $M_{\mathrm{BH}}$ and $M_{\mathrm{NC}}$. We discuss the features we see in these figures. Two possible conclusions emerge from our discussion.

(1) In the $M_{\mathrm{BH}}$-Sersic $n$ figure, those galaxies that lie on the relation seem to prove that there is a relation between $M_{\mathrm{BH}}$ and the morphological transformation of their host galaxies. A few notable outliers are dwarf elliptical galaxies, where the morphological transformation process does not seem to be associate with $\mathrm{BH}$ growth. We speculate that this difference may arise from different mechanisms, that is, mergers for high-mass galaxies and harassment for dwarfs.

(2) In the $M_{\mathrm{BH}}-M_{\mathrm{NC}}$ figure, we can clearly distinguish three regimes; NC dominated, $\mathrm{BH}$ dominated, and transition between the two. We speculate that this could imply that BHs are formed in NCs, then start to grow much faster than their host NCs, and, through a transition phase with similar masses for both components, could then ultimately destroy their host through loss cone depletion. 
We expect further progress in the field to arise from better measurements of $\mathrm{BH}$ masses at the low-mass end of the $M_{\mathrm{BH}}$ mass function and from better measurements of NC masses at the high-mass end of the $M_{\mathrm{BH}}$ mass function. In particular, it might be useful for further research in the field if authors attempting to measure black hole masses also stated more clearly what their constraints on the NC mass are. Currently NCs are treated more or less as a nuisance to get rid of, while a clearer assessment of the constraint on their mass would benefit our understanding of the role NCs play in galaxy nuclei.

\section{Acknowledgments}

The authors thank the referee for an extensive report that significantly improved the presentation of the results in this paper, in particular on the NC upper limits. The authors acknowledge the support and hospitality of the ESO Garching office for science during the genesis of this work. N. Neumayer acknowledges support by the DFG cluster of excellence "Origin and Structure of the Universe". This research has made use of the NASA/IPAC Extragalactic Database (NED) which is operated by the Jet Propulsion Laboratory, California Institute of Technology, under contract with the National Aeronautics and Space Administration. They acknowledge the usage of the HyperLeda database (http://leda.univ-lyon1.fr). This work is based in part on observations made with the NASA/ESA Hubble Space Telescope and obtained from the Hubble Legacy Archive, which is a collaboration between the Space Telescope Science Institute (STScI/NASA), the Space Telescope European Coordinating Facility (ST-ECF/ESA), and the Canadian Astronomy Data Centre (CADC/NRC/CSA). This work is based in part on observations made with the Spitzer Space Telescope, which is operated by the Jet Propulsion Laboratory, California Institute of Technology under a contract with NASA. This publication makes use of data products from the Two Micron All Sky Survey, which is a joint project of the University of Massachusetts and the Infrared Processing and Analysis Center/California Institute of Technology, funded by the National Aeronautics and Space Administration and the National Science Foundation. This paper has made use of NASA's Astrophysics Data System Bibliographic Services.

\section{References}

[1] L. Ferrarese and D. Merritt, "A fundamental relation between supermassive black holes and their host galaxies," Astrophysical Journal Letters, vol. 539, no. 1, pp. L9-L12, 2000.

[2] K. Gebhardt, R. Bender, G. Bower et al., "A relationship between nuclear black hole mass and galaxy velocity dispersion," Astrophysical Journal Letters, vol. 539, no. 1, pp. L13L16, 2000.

[3] N. Häring and H.-W. Rix, "On the black hole mass-bulge mass relation," Astrophysical Journal Letters, vol. 604, no. 2, pp. L89-L92, 2004.
[4] C. M. Carollo, M. Stiavelli, and J. Mack, "Spiral galaxies with WFPC2. II. The nuclear properties of 40 objects," Astronomical Journal, vol. 116, no. 1, pp. 68-84, 1998.

[5] P. Côté, S. Piatek, L. Ferrarese et al., "The ACS virgo cluster survey. VIII. The nuclei of early-type galaxies," Astrophysical Journal, vol. 165, no. 1, pp. 57-94, 2006.

[6] T. Böker, S. Laine, R. P. Van Der Marel et al., "A Hubble Space Telescope census of nuclear star clusters in late-type spiral galaxies. I. Observations and image analysis," Astronomical Journal, vol. 123, no. 3, pp. 1389-1410, 2002.

[7] A. W. Graham, C. A. Onken, E. Athanassoula, and F. Combes, "An expanded $\mathrm{M}_{b h^{-}} \sigma$ diagram, and a new calibration of active galactic nuclei masses," Monthly Notices of the Royal Astronomical Society, vol. 412, no. 4, pp. 2211-2228, 2011.

[8] T. Böker, M. Sarzi, D. E. Mclaughlin et al., "A Hubble Space Telescope census of nuclear star clusters in late-type spiral galaxies. II. Cluster sizes and structural parameter correlations," Astronomical Journal, vol. 127, no. 1, pp. 105$118,2004$.

[9] C. J. Walcher, R. P. Van Der Marel, D. Mclaughlin et al., "Masses of star clusters in the nuclei of bulgeless spiral galaxies," Astrophysical Journal, vol. 618, no. 1, pp. 237-246, 2005.

[10] J. Rossa, R. P. Van Der Marel, T. Böker et al., "Hubble Space Telescope stis spectra of nuclear star clusters in spiral galaxies: dependence of age and mass on hubble type," Astronomical Journal, vol. 132, no. 3, pp. 1074-1099, 2006.

[11] A. C. Seth, J. J. Dalcanton, P. W. Hodge, and V. P. Debattista, "Clues to nuclear star cluster formation from edge-on spirals," Astronomical Journal, vol. 132, no. 6, pp. 2539-2555, 2006.

[12] C. J. Walcher, T. Böker, S. Charlot et al., "Stellar populations in the nuclei of late-type spiral galaxies," Astrophysical Journal, vol. 649, no. 2, pp. 692-708, 2006.

[13] B. Binggeli, F. Barazza, and H. Jerjen, "Off-center nuclei in dwarf elliptical galaxies," Astronomy and Astrophysics, vol. 359, no. 2, pp. 447-456, 2000.

[14] N. Neumayer, C. J. Walcher, D. Andersen, S. F. Sánchez, T. Böker, and H. -W. Rix, "Two-dimensional $\mathrm{H} \alpha$ kinematics of bulgeless disc galaxies," Monthly Notices of the Royal Astronomical Society, vol. 413, no. 3, pp. 1875-1888, 2011.

[15] L. Ferrarese, P. Côté, E. Dalla Bontà et al., "A fundamental relation between compact stellar nuclei, supermassive black holes, and their host galaxies," Astrophysical Journal Letters, vol. 644, no. 1, pp. L21-L24, 2006.

[16] E. H. Wehner and W. E. Harris, "Supermassive black holes to dwarf elliptical nuclei: a mass continuum," Astrophysical Journal Letters, vol. 644, no. 1, pp. L17-L20, 2006.

[17] B. G. Elmegreen, F. Bournaud, and D. M. Elmegreen, "Nuclear black hole formation in clumpy galaxies at high redshift," Astrophysical Journal Letters, vol. 684, no. 2, pp. 829-834, 2008.

[18] J. A. Regan and M. G. Haehnelt, "Pathways to massive black holes and compact star clusters in pre-galactic dark matter haloes with virial temperatures $\gtrsim 10000 \mathrm{~K}$," Monthly Notices of the Royal Astronomical Society, vol. 396, no. 1, pp. 343-353, 2009.

[19] L. Mayer, S. Kazantzidis, A. Escala, and S. Callegari, "Direct formation of supermassive black holes via multi-scale gas inflows in galaxy mergers," Nature, vol. 466, no. 7310, pp. 1082-1083, 2010.

[20] V. Bromm and N. Yoshida, "The first galaxies," Annual Review of Astronomy and Astrophysics, vol. 49, no. 1, pp. 373407, 2011. 
[21] M. Hartmann, V. P. Debattista, A. Seth, M. Cappellari, and T. R. Quinn, "Constraining the role of star cluster mergers in nuclear cluster formation: simulations confront integral-field data," Monthly Notices of the Royal Astronomical Society, vol. 418, no. 4, pp. 2697-2714, 2011.

[22] T. Ebisuzaki, J. Makino, and T. G. Tsuru, "Missing link found? The "runaway" path to supermassive black holes," Astrophysical Journal Letters, vol. 562, no. 1, pp. L19-L22, 2001.

[23] S. F. Portegies Zwart, H. Baumgardt, P. Hut, J. Makino, and S. L.W. McMillan, "Formation of massive black holes through runaway collisions in dense young star clusters," Nature, vol. 428, no. 6984, pp. 724-726, 2004.

[24] M. A. Gürkan, M. Freitag, and F. A. Rasio, "Formation of massive black holes in dense star clusters. I. Mass segregation and core collapse," Astrophysical Journal Letters, vol. 604, no. 2, pp. 632-652, 2004.

[25] M. Freitag, F. A. Rasio, and H. Baumgardt, "Runaway collisions in young star clusters-I. Methods and tests," Monthly Notices of the Royal Astronomical Society, vol. 368, no. 1, pp. 121-140, 2006.

[26] E. Gaburov, A. Gualandris, and S. Portegies Zwart, "On the onset of runaway stellar collisions in dense star clusters-I. Dynamics of the first collision," Monthly Notices of the Royal Astronomical Society, vol. 384, no. 1, pp. 376-385, 2008.

[27] E. Glebbeek, E. Gaburov, S. E. De Mink, O. R. Pols, and S. F.P. Zwart, "The evolution of runaway stellar collision products," Astronomy and Astrophysics, vol. 497, no. 1, pp. 255-264, 2009.

[28] A. V. Filippenko and L. C. Ho, "A low-mass central black hole in the bulgeless Seyfert 1 galaxy NGC 4395," Astrophysical Journal Letters, vol. 588, no. 1, pp. L13-L16, 2003.

[29] A. Seth, M. Agüeros, D. Lee, and A. Basu-Zych, "The coincidence of nuclear star clusters and active galactic nuclei," Astrophysical Journal Letters, vol. 678, no. 1, pp. 116-130, 2008.

[30] R. Schödel, A. Eckart, T. Alexander et al., "The structure of the nuclear stellar cluster of the Milky Way," Astronomy and Astrophysics, vol. 469, no. 1, pp. 125-146, 2007.

[31] R. Genzel, F. Eisenhauer, and S. Gillessen, "The Galactic center massive black hole and nuclear star cluster," Reviews of Modern Physics, vol. 82, no. 4, pp. 3121-3195, 2010.

[32] M. Freitag, M. Atakan Gürkan, and F. A. Rasio, "Runaway collisions in young star clusters-II. Numerical results," Monthly Notices of the Royal Astronomical Society, vol. 368, no. 1, pp. 141-161, 2006.

[33] A. W. Graham and S. P. Driver, "A log-quadratic relation for predicting supermassive black hole masses from the host bulge sérsic index," Astrophysical Journal Letters, vol. 655, no. 1, pp. 77-87, 2007.

[34] J. E. Greene, C. Y. Peng, M. Kim et al., "Precise black hole masses from megamaser disks: black hole-bulge relations at low mass," Astrophysical Journal Letters, vol. 721, no. 1, pp. 26-45, 2010.

[35] A. V. Filippenko and W. L. W. Sargent, "Discovery of an extremely low luminosity Seyfert 1 nucleus in the dwarf galaxy NGC 4395," Astrophysical Journal, vol. 342, pp. L11L14, 1989.

[36] J. C. Shields, C. J. Walcher, T. Böker, L. C. Ho, H. -W. Rix, and R. P. Van Der Marel, "An accreting black hole in the nuclear star cluster of the bulgeless galaxy NGC 1042," Astrophysical Journal Letters, vol. 682, no. 1, pp. 104-109, 2008.

[37] A. J. Barth, L. E. Strigari, M. C. Bentz, J. E. Greene, and L. C. Ho, "Dynamical constraints on the masses of the nuclear star cluster and black hole in the late-type spiral galaxy NGC 3621," Astrophysical Journal Letters, vol. 690, no. 1, pp. 10311044, 2009.

[38] M. Gliozzi, S. Satyapal, M. Eracleous, L. Titarchuk, and C. C. Cheung, "A chandra view of NGC 3621: a bulgeless galaxy hosting an agn in its early phase?" Astrophysical Journal Letters, vol. 700, no. 2, pp. 1759-1767, 2009.

[39] S. Satyapal, D. Vega, R. P. Dudik, N. P. Abel, and T. Heckman, "Spitzer uncovers active galactic nuclei missed by optical surveys in seven late-type galaxies," Astrophysical Journal Letters, vol. 677, no. 2, pp. 926-942, 2008.

[40] J. E. Greene and L. C. Ho, "A new sample of low-mass black holes in active galaxies," Astrophysical Journal Letters, vol. 670, no. 1, pp. 92-104, 2007.

[41] A. J. Barth, J. E. Greene, and L. C. Ho, "Low-mass seyfert 2 galaxies in the sloan digital sky survey," Astronomical Journal, vol. 136, no. 3, pp. 1179-1200, 2008.

[42] W. McAlpine, S. Satyapal, M. Gliozzi, C. C. Cheung, R. M. Sambruna, and M. Eracleous, "Black holes in bulgeless galaxies: an XMM-Newton investigation of NGC 3367 and NGC 4536," Astrophysical Journal Letters, vol. 728, article 25, 2011.

[43] K. Gebhardt, T. R. Lauer, J. Kormendy et al., "M33: a galaxy with no supermassive black hole," Astronomical Journal, vol. 122, no. 5, pp. 2469-2476, 2001.

[44] D. Merritt, L. Ferrarese, and C. L. Joseph, "No supermassive black hole in M33?” Science, vol. 293, no. 5532, pp. 11161118, 2001.

[45] P. Erwin and D. Gadotti, "Do nuclear star clusters and supermassive black holes follow the same host-galaxy correlations?" in Proceedings of the American Institute of Physics Conference, V. P. Debattista and C. C. Popescu, Eds., vol. 1240, pp. 223-226, 2010.

[46] J. Kormendy, R. Bender, and M. E. Cornell, "Supermassive black holes do not correlate with galaxy disks or pseudobulges," Nature, vol. 469, no. 7330, pp. 374-376, 2011.

[47] M. Volonteri, G. Lodato, and P. Natarajan, "The evolution of massive black hole seeds," Monthly Notices of the Royal Astronomical Society, vol. 383, no. 3, pp. 1079-1088, 2008.

[48] T. Di Matteo, V. Springel, and L. Hernquist, "Energy input from quasars regulates the growth and activity of black holes and their host galaxies," Nature, vol. 433, no. 7026, pp. 604607, 2005.

[49] P. F. Hopkins, L. Hernquist, T. J. Cox, T. Di Matteo, B. Robertson, and V. Springel, "A unified, merger-driven model of the origin of starbursts, quasars, the cosmic X-ray background, supermassive black holes, and galaxy spheroids," Astrophysical Journal, vol. 163, no. 1, pp. 1-49, 2006.

[50] C. Y. Peng, "How mergers may affect the mass scaling relation between geuvitationally bound systems," Astrophysical Journal Letters, vol. 671, no. 2, pp. 1098-1107, 2007.

[51] E. Noyola, K. Gebhardt, M. Kissler-Patig et al., "Very large telescope kinematics for omega Centauri: further support for a central black hole," Astrophysical Journal Letters, vol. 719, no. 1, pp. L60-L64, 2010.

[52] K. Jahnke and A. Macciò, "The Non-causal Origin of the Black-hole-galaxy Scaling Relations," The Astrophysical Journal, vol. 734, no. 2, article 92, 2011.

[53] E. Emsellem, G. Monnet, and R. Bacon, "The multi-gaussian expansion method: a tool for building realistic photometric and kinematical models of stellar systems I. The formalism," Astronomy and Astrophysics, vol. 285, pp. 723-738, 1994.

[54] M. Cappellari, "Efficient multi-Gaussian expansion of galaxies," Monthly Notices of the Royal Astronomical Society, vol. 333, no. 2, pp. 400-410, 2002. 
[55] J. Krist, Astronomical Data Analysis Software and Systems IV, vol. 77 of Astronomical Society of the Pacific Conference Series, 1995, edited by R. A. Shaw, H. E. Payne and J. J. E. Hayes.

[56] M. Cappellari, "Measuring the inclination and mass-to-light ratio of axisymmetric galaxies via anisotropic Jeans models of stellar kinematics," Monthly Notices of the Royal Astronomical Society, vol. 390, no. 1, pp. 71-86, 2008.

[57] P. Serra and S. C. Trager, "On the interpretation of the age and chemical composition of composite stellar populations determined with line-strength indices," Monthly Notices of the Royal Astronomical Society, vol. 374, no. 3, pp. 769-774, 2007.

[58] K. Gültekin, D. O. Richstone, K. Gebhardt et al., "The $M-\sigma$ and $M-L$ relations in galactic bulges, and determinations of their intrinsic scatter," Astrophysical Journal Letters, vol. 698, no. 1, pp. 198-221, 2009.

[59] B. M. Peterson, M. C. Bentz, L. -B. Desroches et al., "Multiwavelength monitoring of the dwarf seyfert 1 galaxy NGC 4395. I. A reverberation-based measurement of the black hole mass," Astrophysical Journal, vol. 632, no. 2, pp. 799-808, 2005.

[60] A. J. Barth, L. C. Ho, R. E. Rutledge, and W. L.W. Sargent, "POX 52: a dwarf Seyfert 1 galaxy with an intermediate-mass black hole," Astrophysical Journal Letters, vol. 607, no. 1, pp. 90-102, 2004.

[61] M. Valluri, L. Ferrarese, D. Merritt, and C. L. Joseph, "The low end of the supermassive black hole mass function: constraining the mass of a nuclear black hole in NGC 205 via stellar kinematics," Astrophysical Journal, vol. 628, no. 1, pp. 137-152, 2005.

[62] T. Böker, R. P. Van Der Marel, and W. D. Vacca, "CO band head spectroscopy of IC 342: mass and age of the nuclear star cluster 1," Astronomical Journal, vol. 118, no. 2, pp. 831-842, 1999.

[63] A. C. Seth, M. Cappellari, N. Neumayer et al., "The NGC 404 nucleus: star cluster and possible intermediate-mass black hole," Astrophysical Journal Letters, vol. 714, no. 1, pp. 713$731,2010$.

[64] K. Gebhardt, R. M. Rich, and C. H.O. Luis, "An intermediatemass black hole in the globular cluster G1: improved significance from New Keck and Hubble Space Telescope observations," Astrophysical Journal, vol. 634, no. 2, pp. $1093-$ 1102, 2005.

[65] E. Noyola, K. Gebhardt, and M. Bergmann, "Gemini and Hubble Space Telescope evidence for an intermediate-mass black hole in $\omega$ Centauri," Astrophysical Journal Letters, vol. 676, no. 2, pp. 1008-1015, 2008.

[66] B. Jalali, H. Baumgardt, M. Kissler-Patig et al., "A Dynamical N-body Model for the Central Region of $\omega$ Centauri," http://adsabs.harvard.edu/abs/2011arXiv1111.5011J .

[67] N. Lützgendorf, M. Kissler-Patig, E. Noyola et al., "Kinematic signature of an intermediate-mass black hole in the globular cluster NGC 6388," Astronomy \& Astrophysics, vol. 533, artilce A36, 2011.

[68] J. Anderson and R. P. Van Der Marel, "New limits on an intermediate-mass black hole in omega centauri. I. Hubble Space Telescope photometry and proper motions," Astrophysical Journal Letters, vol. 710, no. 2, pp. 1032-1062, 2010.

[69] R. P. Van Der Marel and J. Anderson, "New limits on an intermediate-mass black hole in omega centauri. II. Dynamical models," Astrophysical Journal Letters, vol. 710, no. 2, pp. 1063-1088, 2010.

[70] H. Baumgardt, J. Makino, H. U. T. Piet, S. McMillan, and S. P. Zwart, "A dynamical model for the globular cluster G1,"
Astrophysical Journal Letters, vol. 589, no. 1, pp. L25-L28, 2003.

[71] J. E. Greene, L. C. Ho, and A. J. Barth, "Black holes in pseudobulges and spheroidals: a change in the black holebulge scaling relations at low mass," Astrophysical Journal Letters, vol. 688, no. 1, pp. 159-179, 2008.

[72] K. Ganda, R. F. Peletier, M. Balcells, and J. Falcón-Barroso, "The nature of late-type spiral galaxies: structural parameters, optical and near-infrared colour profiles and dust extinction," Monthly Notices of the Royal Astronomical Society, vol. 395, no. 3, pp. 1669-1694, 2009.

[73] T. Weinzirl, S. Jogee, S. Khochfar, A. Burkert, and J. Kormendy, "Bulge $n$ and $B / T$ in high-mass galaxies: constraints on the origin of bulges in hierarchical models," Astrophysical Journal Letters, vol. 696, no. 1, pp. 411-447, 2009.

[74] C. Y. Peng, L. C. Ho, C. D. Impey, and H.-W. Rix, "Detailed structural decomposition of galaxy images," Astronomical Journal, vol. 124, no. 1, pp. 266-293, 2002.

[75] A. W. Graham and L. R. Spitler, "Quantifying the coexistence of massive black holes and dense nuclear star clusters," Monthly Notices of the Royal Astronomical Society, vol. 397, no. 4, pp. 2148-2162, 2009.

[76] K. Gültekin, S. Tremaine, A. Loeb, and D. O. Richstone, "Observational Selection Effects and the M- $\sigma$ Relation," The Astrophysical Journal, vol. 738, no. 1, article 17, 2011.

[77] C. Scorza and F. C. Van Den Bosch, "Nuclear stellar discs in early-type galaxies-II. Photometric properties," Monthly Notices of the Royal Astronomical Society, vol. 300, no. 2, pp. 469-478, 1998.

[78] K. Gebhardt and J. Thomas, "The black hole mass, stellar mass-to-light ratio, and Dark Halo in m87," Astrophysical Journal Letters, vol. 700, no. 2, pp. 1690-1701, 2009.

[79] P. J. Young, J. A. Westphal, J. Kristian, C. P. Wilson, and F. P. Landauer, "Evidence for a supermassive object in the nucleus of the galaxy M87 from SIT and CCD area photometry," Astrophysical Journal, vol. 221, pp. 721-730, 1978.

[80] G. A. Bower, R. F. Green, A. C. Quillen et al., "The ionization source in the nucleus of M84," Astrophysical Journal Letters, vol. 534, no. 1, pp. 189-200, 2000.

[81] J. L. Walsh, A. J. Barth, and M. Sarzi, “The supermassive black hole in M84 revisited," Astrophysical Journal Letters, vol. 721, no. 1, pp. 762-776, 2010.

[82] L. Ferrarese, H. C. Ford, and W. Jaffe, "Evidence for a massive black hole in the active galaxy NGC 4261 from bubble space telescope images and spectra," Astrophysical Journal Letters, vol. 470, no. 1, pp. 444-459, 1996.

[83] S. P. Rusli, J. Thomas, P. Erwin, R. P. Saglia, N. Nowak, and R. Bender, "The central black hole mass of the high- $\sigma$ but low-bulge-luminosity lenticular galaxy NGC 1332," Monthly Notices of the Royal Astronomical Society, vol. 410, no. 2, pp. 1223-1236, 2011.

[84] N. Devereux, H. Ford, Z. Tsvetanov, and G. Jacoby, "STIS spectroscopy of the central 10 parsecs of M81: evidence for a massive black hole," Astronomical Journal, vol. 125, no. 3, pp. 1226-1235, 2003.

[85] M. J. Jee, J. P. Blakeslee, M. Sirianni, A. R. Martel, R. L. White, and H. C. Ford, "Principal component analysis of the time- and position-dependent point-spread function of the advanced camera for surveys," Publications of the Astronomical Society of the Pacific, vol. 119, no. 862, pp. 14031409, 2007.

[86] L. Ferrarese, P. Côté, A. Jordán et al., "The ACS Virgo Cluster survey. VI. Isophotal analysis and the structure of early-type 
galaxies," Astrophysical Journal, Supplement Series, vol. 164, no. 2, pp. 334-434, 2006.

[87] T. R. Lauer, K. Gebhardt, S. M. Faber et al., "The centers of early-type galaxies with Hubble Space Telescope. VI. Bimodal central surface brightness profiles," Astrophysical Journal, vol. 664, no. 1, pp. 226-256, 2007.

[88] G. De Francesco, A. Capetti, and A. Marconi, "Measuring supermassive black holes with gas kinematics: the active so galaxy NGC 3998," Astronomy and Astrophysics, vol. 460, no. 2, pp. 439-448, 2006.

[89] N. Cretton and F. C. Van Den Bosch, "Evidence for a massive black hole in the S0 galaxy NGC 4342," Astrophysical Journal Letters, vol. 514, no. 2, pp. 704-724, 1999.

[90] K. Gebhardt, D. Richstone, J. Kormendy et al., "Axisymmetric, three-integral models of galaxies: a massive black hole in NGC 3379," Astronomical Journal, vol. 119, no. 3, pp. 1157$1171,2000$.

[91] D. Merritt and A. Szell, "Dynamical cusp regeneration," Astrophysical Journal, vol. 648, no. 2, pp. 890-899, 2006.

[92] M. Koleva, P. Prugniel, S. De Rijcke, and W. W. Zeilinger, "Age and metallicity gradients in early-type galaxies: a dwarfto-giant sequence," Monthly Notices of the Royal Astronomical Society, vol. 417, no. 3, pp. 1643-1671, 2011.

[93] H. Zinnecker, C. J. Keable, J. S. Dunlop, R. D. Cannon, and W. K. Griths, "The harlow-shapley symposium on globular cluster systems in galaxies," in Proceedings of the 126th Symposium of the International Astronomical Union (IAU '88), J. E. Grindlay and A. G. D. Philip, Eds., vol. 126, p. 603, 1988.

[94] K. C. Freeman, The Globular Cluster-Galaxy Connection, vol. 48 of Astronomical Society of the Pacific Conference Series, 1993, edited by G. H. Smith and J. P. Brodie.

[95] K. Bekki and K. C. Freeman, "Formation of $\omega$ Centauri from an ancient nucleated dwarf galaxy in the young Galactic disc," Monthly Notices of the Royal Astronomical Society, vol. 346, no. 2, pp. L11-L15, 2003.

[96] T. Böker, "Are globular clusters the remnant nuclei of progenitor disk galaxies?" Astrophysical Journal Letters, vol. 672, no. 2, pp. L111-L114, 2008.

[97] M. Milosavljević, "On the origin of nuclear star clusters in late-type spiral galaxies," Astrophysical Journal Letters, vol. 605, no. 1, pp. L13-L16, 2004.

[98] K. Bekki, W. J. Couch, and Y. Shioya, "Dissipative transformation of nonnucleated dwarf galaxies into nucleated systems," Astrophysical Journal Letters, vol. 642, no. 2, pp. L133-L136, 2006.

[99] J. Pflamm-Altenburg and P. Kroupa, "Recurrent gas accretion by massive star clusters, multiple stellar populations and mass thresholds for spheroidal stellar systems," Monthly Notices of the Royal Astronomical Society, vol. 397, no. 1, pp. 488-494, 2009.

[100] S. D. Tremaine, J. P. Ostriker, and L. Spitzer, "The formation of the nuclei of galaxies. I-M31," Astrophysical Journal, vol. 196, pp. 407-411, 1975.

[101] R. Capuzzo-Dolcetta, "The evolution of the globular cluster system in a triaxial galaxy: can a galactic nucleus form by globular cluster capture?" Astrophysical Journal Letters, vol. 415, no. 2, pp. 616-630, 1993.

[102] D. E. Mclaughlin, "Was the compact nucleus in M87 formed by destroyed globular clusters?" Astronomical Journal, vol. 109, no. 5, pp. 2034-2037, 1995.

[103] K. Bekki, W. J. Couch, M. J. Drinkwater, and Y. Shioya, "Cluster cannibalism and scaling relations of galactic stellar nuclei," Astrophysical Journal Letters, vol. 610, no. 1, pp. L13L16, 2004.

[104] P. Miocchi, R. C. Dolcetta, P. Di Matteo, and A. Vicari, "Merging of globular clusters in inner galactic regions. I. Do they survive the tidal interaction?" Astrophysical Journal Letters, vol. 644, no. 2, pp. 940-953, 2006.

[105] M. Agarwal and M. Milosavljević, "Nuclear star clusters from clustered star formation," Astrophysical Journal Letters, vol. 729, article 35, 2011.

[106] S. Nayakshin, M. I. Wilkinson, and A. King, "Competitive feedback in galaxy formation," Monthly Notices of the Royal Astronomical Society, vol. 398, no. 1, pp. L54-L57, 2009.

[107] D. Merritt, "Dynamics of galaxy cores and supermassive black holes," Reports on Progress in Physics, vol. 69, no. 9, pp. 2513-2579, 2006.

[108] K. Bekki and A. W. Graham, "On the transition from nuclearcluster- to black-hole-dominated galaxy cores," Astrophysical Journal Letters, vol. 714, no. 2, pp. L313-L317, 2010.

[109] K. Gebhardt, J. Adams, D. Richstone et al., "The black hole mass in M87 from Gemini/NIFS adaptive optics observations," Astrophysical Journal Letters, vol. 729, article 119, 2011.

[110] G. A. Bower, R. F. Green, A. Danks et al., "Kinematics of the nuclear ionized gas in the radio galaxy M84 (NGC 4374)," Astrophysical Journal Letters, vol. 492, no. 2, pp. L111-L114, 1998.

[111] K. Gebhardt, D. Richstone, S. Tremaine et al., "Axisymmetric dynamical models of the central regions of galaxies," Astrophysical Journal Letters, vol. 583, no. 1, pp. 92-115, 2003.

[112] M. Sarzi, H.-W. Rix, J. C. Shields et al., "Supermassive black holes in bulges," Astrophysical Journal Letters, vol. 550, no. 1, pp. 65-74, 2001.

[113] K. L. Shapiro, M. Cappellari, T. De Zeeuw et al., "The black hole in NGC 3379: a comparison of gas and stellar dynamical mass measurements with HST and integral-field data," Monthly Notices of the Royal Astronomical Society, vol. 370, no. 2, pp. 559-579, 2006. 

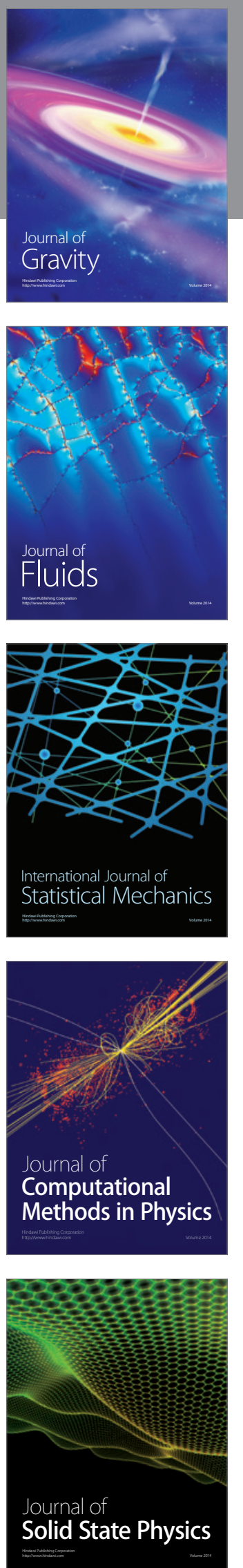

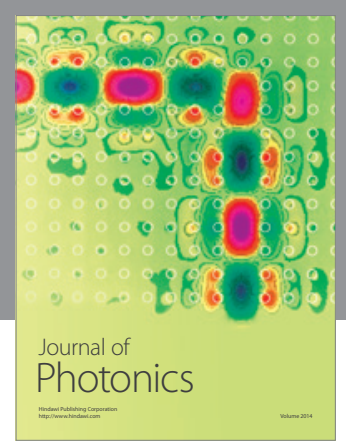

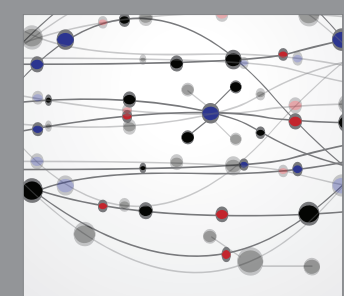

The Scientific World Journal
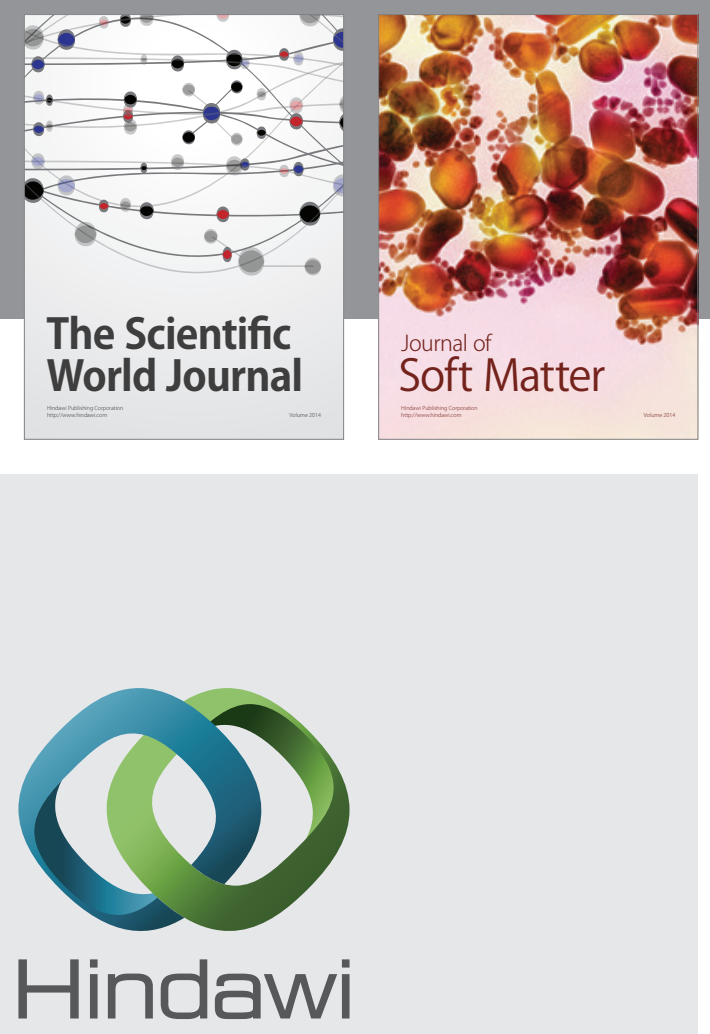

Submit your manuscripts at

http://www.hindawi.com
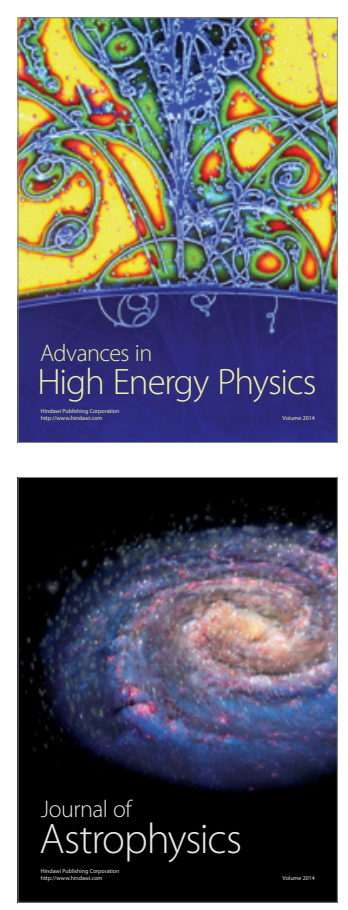
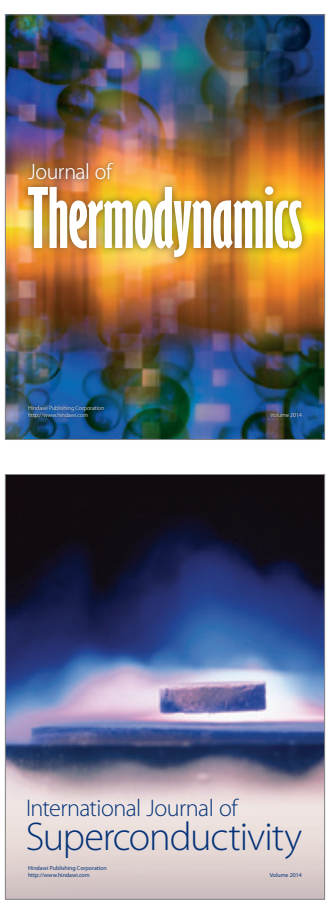
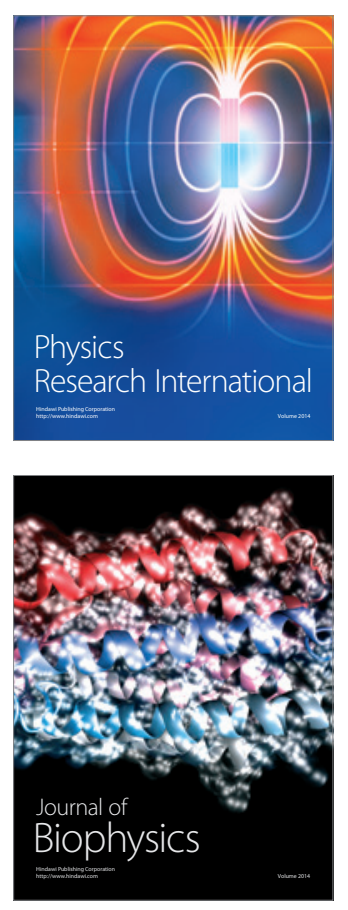
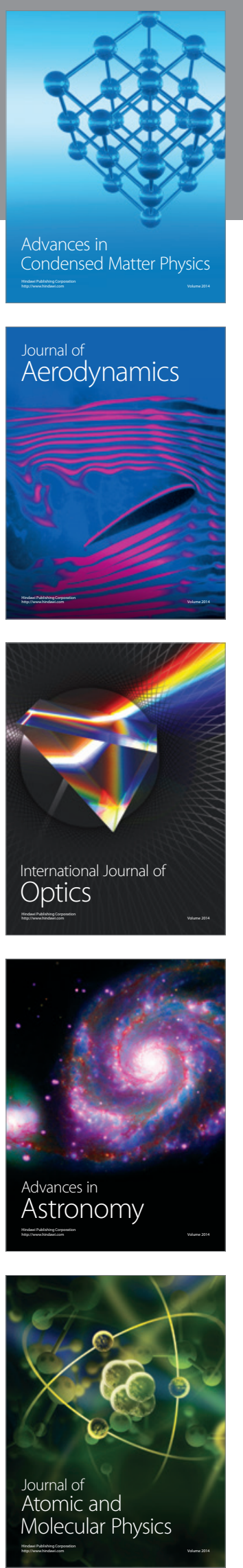